(C) 2017 IEEE. Personal use of this material is permitted. Permission from IEEE must be obtained for all other uses, in any current or future media, including reprinting/republishing this material for advertising or promotional purposes, creating new collective works, for resale or redistribution to servers or lists, or reuse of any copyrighted component of this work in other works. 


\title{
Predictive Control of Cascaded H-Bridge Converters Under Unbalanced Power Generation
}

\author{
Ricardo P. Aguilera, Member, IEEE, Pablo Acuna, Member, IEEE, Yifan Yu, Student Member, IEEE, \\ Georgios Konstantinou, Member, IEEE, Christopher D. Townsend, Member, IEEE, Bin Wu, Fellow, IEEE \\ and Vassilios G. Agelidis, Fellow, IEEE
}

\begin{abstract}
This work presents a predictive control strategy for grid-connected Cascaded H-Bridge (CHB) converters under unbalanced power generation among each converter phase. The proposed controller belongs to the Finite-Control-Set Model Predictive Control (FCS-MPC) family and is designed to extract unbalanced power from each CHB converter phase while providing balanced power to the grid. The key novelty of this strategy lies in the way the unbalanced power generation among the phases is explicitly considered into the optimal control problem. Power balance is achieved by enforcing the CHB converter to work with a suitable zero-sequence voltage component. The proposed predictive controller is directly formulated in the original $a b c$-framework to account for the commonmode voltage. Simulation and experimental results are provided to verify the effectiveness of the proposed FCS-MPC strategy.
\end{abstract}

Index Terms-Multilevel converters, cascaded H-bridge converters, DC-AC power converters, finite control set, model predictive control, controller performance, power quality, photovoltaics, smart grids.

\section{INTRODUCTION}

$\mathbf{M}$ ULTILEVEL Converters (MCs) are an interesting technology for medium/high-voltage and high power applications [1]. Despite the fact that several MC topologies have been proposed in the literature, they all present a common feature: the ability to produce high quality voltage and current waveforms at medium/high-voltage range by using power switches rated at lower voltage values. To distribute the total voltage among the internal power switches, special modulation and/or control techniques are used. Thus, MCs are able to provide a staircase output voltage with reduced $d v / d t$ and low harmonic distortion. This has allowed MCs to be used

Manuscript received June 26, 2015; revised November 26, 2015, March 3, 2016, April 14, 2016, and June 2, 2016; accepted June 21, 2016.

R. P. Aguilera is with The School of Electrical, Mechanical and Mechatronic Systems, University of Technology Sydney (UTS), NSW 2007, Australia. (email: raguilera@ieee.org).

P. Acuna, Y. Yu, G. Konstantinou and V. G. Agelidis are with The University of New South Wales, Sydney, NSW 2052, Australia. (email: pablo.acuna@unsw.edu.au).

C. Townsend is with the School of Electrical Engineering and Computer Science, The University of Newcastle, Callaghan, NSW 2308, Australia. (email: townsend@ieee.org).

B. Wu is with the Department of Electrical and Computer Engineering, Ryerson University, Canada (e-mail: bwu@ee.ryerson.ca). in several high power applications, such as high-power drives [2], active power filters [3], renewable energy [4], etc.

Among the different MCs one can find in the literature, the most popular and widely used are: the Neutral Point Clamped (NPC) [3], Flying Capacitor (FC) [5], Cascaded HBridge (CHB) [6], and Modular Multilevel Converter (MMC) [7]. This work is particularly focused on the control of $\mathrm{CHB}$ converters. A distinctive feature of $\mathrm{CHB}$ converters is that they are built upon cells, which comprises multiple isolated dc-dc converters and H-bridges. This makes them particularly suitable for the integration of solar PV plants to the electricity grid, where several PV arrays are separately connected to each cell [4]. Nevertheless, due to the large geographic footprint of PV solar plants, they are likely to be affected by partial shading and dust settlement. Hence, the maximum available power in each PV string may differ. Therefore, a major operational challenge for PV solar plants is to inject balanced power to the grid under unbalanced power generation from each PV string.

In this context, several control strategies have been proposed to govern $\mathrm{CHB}$ converters under unequal power generation among phases [6], [8]-[10]. The control target is to achieve an inter-phase power balance (symmetric sinusoidal currents) as seen by the grid-side under a certain range of unbalanced powers. To achieve this, standard approaches are realized in several stages based on the well-known Voltage Oriented Control (VOC) strategy [6], [8], [9]. Firstly, the converter currents are rotated to a $d q$-framework. Secondly, standard PI controllers are used to track the required $d q$-current references. In a third stage, the $d q$-voltages provided by the controllers are transformed into the original $a b c$-framework obtaining a symmetrical converter voltage reference, $v_{a b c}^{+}$. In a final stage, a suitable zero-sequence component, $v^{0}$, is added to ensure inter-phase power balance [6]. Finally, this reference, $v_{a b c}=v_{a b c}^{+}+v^{0}$, is synthesized by a PWM stage.

In terms of modern control strategies for power converters, Model Predictive Control (MPC) has emerged as a promising alternative to govern power converters [11], [12]. Different predictive control formulations have been proposed to govern power converters, showing that these methods, in general, may outperform standard PWM-based controllers. Due to its flexibility and ability to consider constraints in the control formulation, Finite Control Set MPC (FCS-MPC) is one of the most popular predictive controller for power converters [11], [13]. FCS-MPC directly considers the state of the power 
switches (or voltage levels) in the optimization as input constraints [14]. Thus, no modulation stages are needed. To obtain the optimal solution, one can evaluate all the possible switch combinations in the cost function and then apply the one which minimizes it. Some examples of recent predictive controllers in power electronics can be found in [11]-[18].

FCS-MPC has often been proposed to govern gridconnected power converters due to the fast dynamic response that can be obtained [19]. In [20], the necessity of having two control loops to regulate the $\mathrm{dc}$ - and ac-side of the converter has been addressed using FCS-MPC. Here, the dcvoltage regulation has been explicitly incorporated into the optimal control problem by adding a proper reference design. Furthermore, in [21], the fast dynamic response of FCSMPC is exploited to fulfill low voltage ride-through capability requirements. Despite the advantages that the aforementioned predictive control approaches offer, their control formulation do not take the unbalanced power generation problem into account. Hence, existing FCS-MPC strategies for grid-connected converters have not been designed for use in PV applications.

Motivated by the above, in this work, an FCS-MPC for CHB converters under unbalanced power generation that extracts unbalanced power from each converter phase while providing balanced power to the grid is proposed. The key novelty of this proposal lies in the way the unbalanced power generation issue is explicitly taken into the optimal control problem. To account for the common-mode voltage, the proposed predictive controller is directly formulated in the original $a b c$-framework. This allows the controller to track balanced currents while synthesizing an asymmetric inverter voltage that ensures interphase power balance. Therefore, no online frame-rotations are needed by the predictive controller. Thus, the proposed predictive controller exhibits a simple structure where no frame-rotations and less cascaded control stages are required. To verify the effectiveness and performance of the proposed predictive controller, simulation and experimental results on a three-phase two-cell CHB converter governed by the proposed predictive control strategy are provided.

\section{CHB CONVERTER MOdel}

The CHB converter topology is shown in Fig. 1. This multilevel converter is comprised of a basic unit called cell, which is a simple 3-level HB (3L-HB) converter electrically fed with an isolated dc-source. For the particular problem at hand, each dc-source is comprised of several PV strings as depicted in Fig. 1. Since most of commercial PV modules can withstand no more than $1 \mathrm{kV}$, dc-dc converters with high frequency transformers are adopted as standard solution to provide isolation between the active part and the grounded frame [9]. To extend the output voltage levels, $\eta$-cells can be cascaded in series, obtaining a maximum of $2 \eta+1$ levels.

\section{A. Continuous-Time Model}

Considering a grid-connected CHB converter, by applying simple circuit analysis of the converter topology shown in

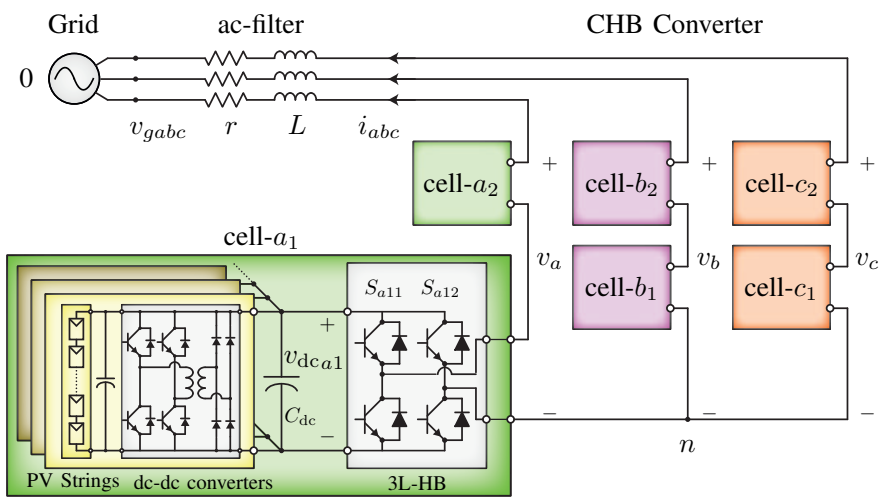

Fig. 1. Circuit diagram of the three-phase two-cell CHB converter.

Fig. 1, the following continuous-time dynamic model for each output current can be obtained:

$$
\frac{d i_{\chi}(t)}{d t}=-\frac{r}{L} i_{\chi}(t)+\frac{1}{L}\left(v_{\chi}(t)-v_{g \chi}(t)-v_{0 n}(t)\right),
$$

for all $\chi \in\{a, b, c\}$. Here, $L$ and $r$ represent the inductance and resistance of the output filter. Additionally, $v_{g \chi}(t)$ stands for the grid voltage per phase, $v_{\chi}(t)$ represents the CHB output voltage per phase, and $v_{0 n}(t)$ is the so-called common-mode voltage, which is given by:

$$
v_{0 n}(t)=\frac{1}{3}\left(v_{a}(t)+v_{b}(t)+v_{c}(t)\right) .
$$

For a generic $\eta$-cell converter, the total CHB output voltage can be expressed in terms of each individual output cell voltage, $v_{\chi j}(t)$, with $j \in\{1, \ldots, \eta\}$, via:

$$
v_{\chi}(t)=\sum_{j=1}^{\eta} v_{\chi j}(t) .
$$

\section{B. Control Input}

In general, when using FCS-MPC for governing power converters, the states of the converter power switches are normally considered as a control input [14]. In this case, the output voltage of each cell can be defined as:

$$
v_{\chi j}=v_{\mathrm{dc} \chi j}\left(S_{\chi j 1}-S_{\chi j 2}\right),
$$

where $v_{\mathrm{dc} \chi j}$ is the isolated dc-voltage at the input of each $3 \mathrm{~L}-$ $\mathrm{HB}$, and $S_{\chi j 1}$ and $S_{\chi j 2}$ are the states of the upper switches, e.g., $S_{a 11}$ and $S_{a 12}$ for cell- $a_{1}$ as shown in Fig. 1. Since each power switch can adopt only two values, i.e., $S_{\chi j 1} \in\{0,1\}$, this results in $2^{2}$ switching combinations per cell. Therefore, taking into account (3), for a three-phase $\eta$-cell CHB converter, the total number of switch combinations is given by:

$$
N_{S C}=2^{6 \eta} \text {. }
$$

Thus, for the case of a two-cell CHB converter, a total of 4096 input (power switch) combinations is obtained. This, in fact, represents a practical implementation problem for the predictive controller, since it is required to evaluate all these input combinations in the cost function to obtain the optimal one. Although powerful processors such as FPGAs and microcontrollers are available to perform these calculations, power 
converters operate with sampling periods $\left(T_{s}\right)$ of approximately $0-200 \mu \mathrm{s}$ [22], [23]. This typically limits the maximum combinations that can be evaluated within $T_{s}$ to a few hundred.

If the execution time $\left(T_{e}\right)$ exceeds $T_{s}$, then the controller will suffer from undesirable task-overrun. To address this issue, one can take advantage of the fact that some of these switch combinations generate the same output voltage per phase. Therefore, this work considers the use of the phase voltage levels, $v_{\ell \chi}$, as control input instead of the power switch states. Thus, the total CHB output voltage in (3) becomes:

$$
v_{\chi}=v_{\mathrm{dc}}^{\star} \cdot v_{\ell \chi}
$$

where $v_{\ell \chi} \in \mathbb{V}=\{-\eta,-\eta+1, \ldots, 0, \ldots, \eta-1, \eta\}$.

Now, for a three-phase $\eta$-cell $\mathrm{CHB}$ converter, the number of voltage level combinations, considering (6), is given by:

$$
N_{V L C}=(2 \eta+1)^{3}
$$

which drastically reduces the input combinations when compared to (5). For example, for the two-cell case, we have now only 125 input (voltage level) combinations.

\section{Discrete-Time Model}

In this work, the implementation of an FCS-MPC strategy for a multilevel CHB converter in the original $a b c$-framework is proposed. To achieve this, the system state, at each discrete instant $k$, is chosen as:

$$
x(k)=i_{a b}(k)=\left[\begin{array}{c}
i_{a}(k) \\
i_{b}(k)
\end{array}\right],
$$

where $i_{c}(k)=-\left(i_{a}(k)+i_{b}(k)\right)$. Then, as previously mentioned, the control input is chosen as:

$$
u(k)=\left[\begin{array}{l}
v_{\ell a}(k) \\
v_{\ell b}(k) \\
v_{\ell c}(k)
\end{array}\right] \in \mathbb{U},
$$

which belongs to the finite control set:

$$
\mathbb{U}=\mathbb{V}^{3}
$$

Therefore, by applying the forward Euler discretization to (1), the following discrete-time dynamic model can be obtained:

$$
i_{a b}(k+1)=A i_{a b}(k)+B u(k)+E v_{g}(k),
$$

where

$$
\begin{aligned}
A & =\left[\begin{array}{cc}
1-\frac{r}{L} T_{s} & 0 \\
0 & 1-\frac{r}{L} T_{s}
\end{array}\right], \\
B & =\frac{v_{\mathrm{dc}}^{\star} T_{s}}{3 L}\left[\begin{array}{ccc}
2 & -1 & -1 \\
-1 & 2 & -1
\end{array}\right], \quad E=-\frac{T_{s}}{L}\left[\begin{array}{ll}
1 & 0 \\
0 & 1
\end{array}\right],
\end{aligned}
$$

and $v_{g}=\left[\begin{array}{ll}v_{g a}(k) & v_{g b}(k)\end{array}\right]^{T}$ is the balanced grid voltage vector which satisfies $v_{g c}(k)=-\left(v_{g a}(k)+v_{g b}(k)\right)$. Notice that the common-mode voltage information is preserved in $B$.

\section{FCS-MPC OF A CHB CONVERTER}

In this Section, a general FCS-MPC framework based on [24] is presented. To implement the horizon-one FCS-MPC strategy, a measurement of the system state $x(k)$ is taken and then a cost function is evaluated for each control input element in $\mathbb{U}$ as per (10). Normally, in power electronics, the cost function only penalizes the current tracking error. Then, the control target is to achieve and maintain a steady state reference for the output current, i.e.,:

$$
i_{a b}^{\star}(t)=\left[\begin{array}{c}
I^{\star} \sin (\omega t+\phi) \\
I^{\star} \sin (\omega t-2 \pi / 3+\phi)
\end{array}\right],
$$

where $I^{\star}$ stands for the peak value of the current reference, $\omega$ represents the grid voltage angular frequency, and $\phi$ is the desired current phase angle with respect to the grid voltage.

Thus, the standard horizon-one cost function can be expressed as (see [11]):

$$
J_{\text {std }}(k)=\left\|i_{a b}^{\prime}(k+1)-i_{a b}^{\star}(k+1)\right\|_{2}^{2},
$$

where $i_{a b}^{\prime}(k+1)$ stands for the CHB current predictions and $\|\cdot\|_{2}^{2}$ represents the quadratic Euclidean norm, i.e., $\left\|a-a^{\star}\right\|_{2}^{2}=\left(a_{1}-a_{1}^{\star}\right)^{2}+\ldots+\left(a_{p}-a_{p}^{\star}\right)^{2}$, for a pair of vectors $a, a^{\star} \in \mathbb{R}^{p}$. As shown in [25], considering only the tracking current error in the cost function leads, in general, to a high common-mode voltage since several inputs generate the same output current. To address this problem, in [25], voltage redundancies are eliminated by selecting only the voltage vectors which generate the lowest common-mode voltage. However, this solution limits the ability to use redundancies for inter-cell power balance purposes.

Recently in [24], an FCS-MPC strategy with guaranteed performance has been proposed for power converters. Here, the use of the input tracking error has also been considered into the cost function:

$$
J(k)=\left\|i_{a b}^{\prime}(k+1)-i_{a b}^{\star}(k+1)\right\|_{2}^{2}+\sigma\left\|u^{\prime}(k)-u^{\star}(k)\right\|_{2}^{2},
$$

where $u^{\prime}(k)$ is the tentative input combination that generates the current prediction $i_{a b}^{\prime}(k+1)$, and $u^{\star}(k)$ is the required CHB output voltage to maintain the current reference (13) in steady-state. Here, the weighting factor $\sigma$ allows one to adjust a desired closed-loop performance; see [24]. Notice that when the system state is near its reference, $i_{a b}(k) \approx i_{a b}^{\star}(k)$, the first part of the cost function is almost zero. Therefore, the second term becomes the dominant term that defines the control action. This leads to an optimal control input, $u^{\mathrm{op}}(k)$, that tracks the input reference, $u^{\star}(k)$, during the steady-state. Consequently, the optimal voltage level to be applied by the converter is the one that minimizes the cost function, i.e.,:

$$
u^{\mathrm{op}}(k)=\arg \left\{\min _{u \in \mathbb{U}} J(k)\right\} .
$$

This procedure is repeated at each sampling instant using the latest measurements of the currents and grid voltages.

\section{Voltage Reference Design to Achieve INTER-PHASE POWER BALANCE}

In this section, a converter voltage reference to be included in the proposed cost function is derived. This allows the 
predictive controller to extract different amounts of power from each $\mathrm{CHB}$ converter phase while injecting balanced power to the grid. This case is particularly important for solar PV plants, which may be affected by partial shading, see [6].

\section{A. Symmetric Voltage Reference}

Firstly, the case of equal power generation between phases is analyzed. Here, the aim is to obtain balanced sinusoidal currents, as per (13), while maintaining a minimum commonmode voltage. To achieve this, firstly, the steady-state derivative of the current reference is obtained:

$$
\frac{d i_{\chi}^{\star}(t)}{d t}=\omega I^{\star} \cos \left(\omega t+\theta_{\chi}+\phi\right),
$$

where $\theta_{\chi} \in\{0,-2 \pi / 3,+2 \pi / 3\}$. Then, evaluating the desired steady-state condition in (1), and considering a null commonmode voltage, i.e., $v_{0 N}^{\star}=0$, the required input to keep the symmetric sinusoidal reference in (13) is given by:

$$
\begin{aligned}
v_{\ell \chi}^{\star}(t)=\frac{1}{v_{\mathrm{dc}}^{\star}}( & I^{\star}\left(X_{L} \cos \left(\omega t+\theta_{\chi}+\phi\right)\right. \\
& \left.\left.+r \sin \left(\omega t+\theta_{\chi}+\phi\right)\right)+v_{g \chi}(t)\right),
\end{aligned}
$$

where $X_{L}=\omega L$. Consequently, to achieve balanced sinusoidal currents with a reduced common-mode voltage, the FCS-MPC strategy is implemented by using the proposed cost function, $J(k)$ in (15), with $i_{a b}^{\star}(k)$ as per (13) and

$$
u^{\star}(k)=\left[\begin{array}{c}
v_{\ell a}^{\star}(k) \\
v_{\ell b}^{\star}(k) \\
v_{\ell c}^{\star}(k)
\end{array}\right] .
$$

\section{B. Voltage Reference Under Unbalanced Power Genera- tion}

A standard approach to achieve inter-phase power balance under unbalanced power generation amongst the three phases of the converter is by generating a suitable zero sequence voltage at the $\mathrm{CHB}$ converter output terminals [9], which yields to unbalanced inverter voltages. Thus, the desired voltage reference can be expressed by:

$$
\tilde{v}_{\chi}^{\star}(t)=v_{\chi}^{\star}+(t)+v^{0}(t) .
$$

Now, it is assumed that the nominal power that the power source (e.g., PV solar plant) can provide, in a normal condition, is $p_{3-\phi}$. Then, to account for the reduction in power per phase, an inter-phase power generation ratio $\lambda_{\chi} \in[0,1]$, that represents the maximum achievable power to be extracted, is introduced, i.e.,:

$$
\lambda_{\chi}=\frac{p_{\chi}}{p_{3-\phi} / 3} .
$$

In this work, $\lambda_{\chi}$ is considered to be supplied by the interaccion between a standard Maximum Power Point Tracking (MPPT) algorithm and the power reference generation. On the one hand, the MPPT algorithm governs the isolated dc-dc converter to extract the maximum available power from its PV string by increasing or decreasing the current injected to the dc-link. On the other hand, the external power reference generator is implemented to regulate each isolated dc-voltage, $v_{\mathrm{dc} \chi j}$, to the same targeted reference, $v_{\mathrm{dc}}^{\star}$. Here, a standard control strategy for the dc-dc converter and the power reference generation, based on the well-known VOC with traditional PI controllers, is adopted from [9], which allows one to generate $\lambda_{\chi}$ as per (21). The block diagrams of the isolated dc-dc converter control and the power reference generator are shown in Fig. 2(a) and 2(b) respectively. Therefore, the required balanced current, under an unbalanced power source, can be obtained as per:

$$
I^{\star}=\frac{3}{2} \frac{p_{3-\phi}}{\hat{V}_{g}} \frac{\left(\lambda_{a}+\lambda_{b}+\lambda_{c}\right)}{3} \frac{1}{\cos (\phi)},
$$

where $\hat{V}_{g}$ is the amplitude of the grid voltage and the angle $\phi$ can be obtained from:

$$
\tan (\phi)=\frac{q^{\star}}{p_{3-\phi}\left(\lambda_{a}+\lambda_{b}+\lambda_{c}\right)} .
$$

Consequently, the positive sequence component of the unbalanced CHB voltage reference is:

$$
v_{\chi}^{\star}+(t)=v_{\mathrm{dc}}^{\star} \cdot v_{\ell \chi}^{\star}(t),
$$

where $v_{\ell \chi}^{\star}(t)$ is obtained from (18) by using (22) and (23). Hence, the required zero sequence voltage is given by:

$$
v^{0}(t)=\hat{V}^{0} \sin \left(\omega t+\theta_{0}\right)
$$

where

$$
\begin{aligned}
\hat{V}^{0} & =\frac{\sqrt{6} \Delta}{3\left(\lambda_{a}+\lambda_{b}+\lambda_{c}\right)}\left(\hat{V}_{g} \sqrt{3}\right), \\
\theta_{0} & =\left\{\begin{array}{l}
\sin ^{-1}\left(\frac{\sqrt{6}\left(\lambda_{c}-\lambda_{b}\right)}{2 \Delta}\right) \\
\frac{2 \pi}{3}+\sin ^{-1}\left(\frac{\sqrt{6}\left(\lambda_{b}-\lambda_{a}\right)}{2 \Delta}\right) \\
\frac{4 \pi}{3}+\sin ^{-1}\left(\frac{\sqrt{6}\left(\lambda_{a}-\lambda_{c}\right)}{2 \Delta}\right) \\
\text { Sectors (I), (VI) }
\end{array}\right. \\
\Delta & =\sqrt{\left(\lambda_{a}-\lambda_{b}\right)^{2}+\left(\lambda_{b}-\lambda_{c}\right)^{2}+\left(\lambda_{a}-\lambda_{c}\right)^{2}} .
\end{aligned}
$$

For the sake of brevity, details on how to derive the above zero sequence voltage reference are not included in this paper. However, they can be found in [9].

Consequently, to extract unbalanced power from each $\mathrm{CHB}$ converter phase while injecting balanced power into the grid, the FCS-MPC strategy is implemented by using the proposed cost function, $J(k)$ in (15), with $i_{a b}^{\star}(k)$ as per (13) with (22), and

$$
u^{\star}(k)=\left[\begin{array}{c}
\tilde{v}_{\ell a}^{\star}(k) \\
\tilde{v}_{\ell b}^{\star}(k) \\
\tilde{v}_{\ell c}^{\star}(k)
\end{array}\right],
$$

where

$$
\tilde{v}_{\ell \chi}^{\star}(k)=v_{\ell \chi}^{\star}(k)+\frac{1}{v_{\mathrm{dc}}^{\star}} v^{0}(k) .
$$

Finally, a block diagram of the proposed predictive control strategy is presented in Fig. 2(c).

Notice that for the balanced case when $\lambda_{a}=\lambda_{b}=\lambda_{c}$, the common-mode voltage reference becomes zero, i.e., $v_{0 n}^{\star}=0$. 


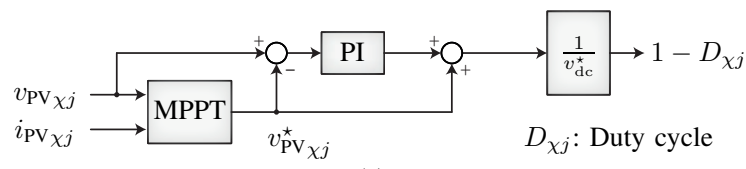

(a)

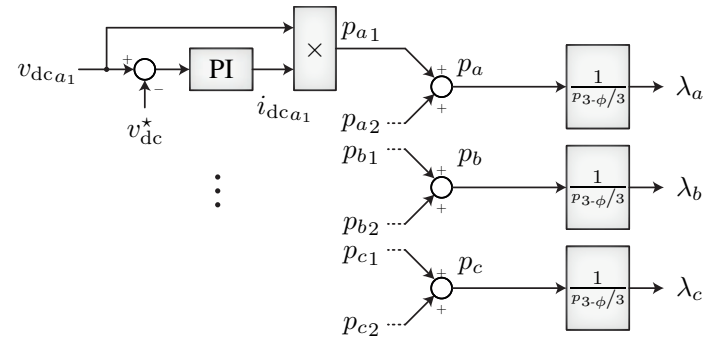

(b)

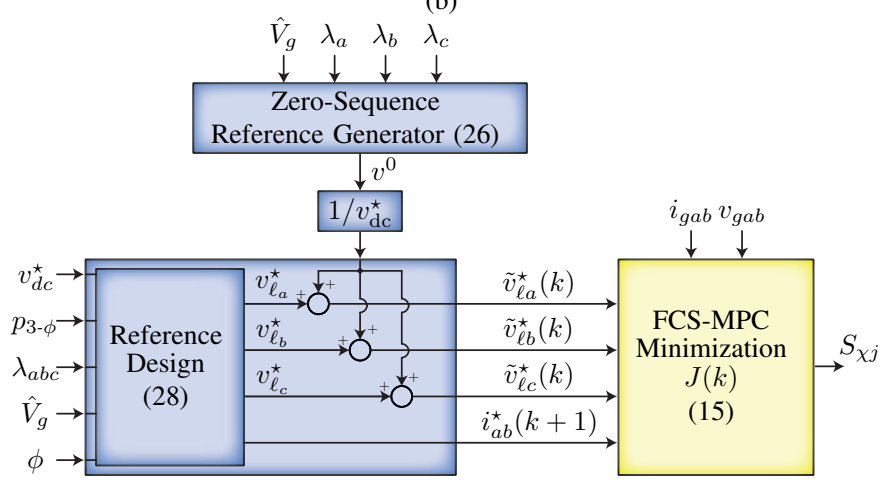

(c)

Fig. 2. Control implementation: (a) Standard control implementation for dc-dc converter, (b) power references generation, and (c) proposed FCS-MPC control diagram with zero-sequence reference generator.

This leads to the symmetric case presented in Section IV-A, i.e., $\tilde{v}_{\ell_{x}}^{\star}=v_{\ell_{x}}^{\star}$. It is important to emphasize that (26) is not the only viable option for the zero-sequence reference $v^{0}$. Any zero-sequence reference that guarantees inter-phase power balance (e.g., [6], [8], [9]) can be applied along with the proposed predictive controller.

\section{Results}

Simulation and experimental results are performed to analyze both the steady-state and dynamic performance of the proposed FCS-MPC strategy under unbalanced power generation. Moreover, these experimental results are compared with a set of results that utilize a PWM-based PI controller. Unless otherwise stated, the results here presented were obtained considering a unity power factor.

\section{A. Simulation Results}

Simulation results of a three-phase two-cell CHB converter governed by the proposed FCS-MPC strategy are presented in this section. The results have been obtained by means of MATLAB-Simulink and PLECS. The main system parameters are presented in Table I.

1) Simulation-based Sensitivity Analysis: Figure 3 shows the steady-state performance of the proposed control strategy, in terms of common-mode voltage, for different values of the weighting factor $\sigma$. According to Fig. 3, a suitable value for $\sigma$, which results in an average value close to zero, $\overline{v_{0 n}} \approx 0$,
TABLE I

SYSTEM PARAMETERS

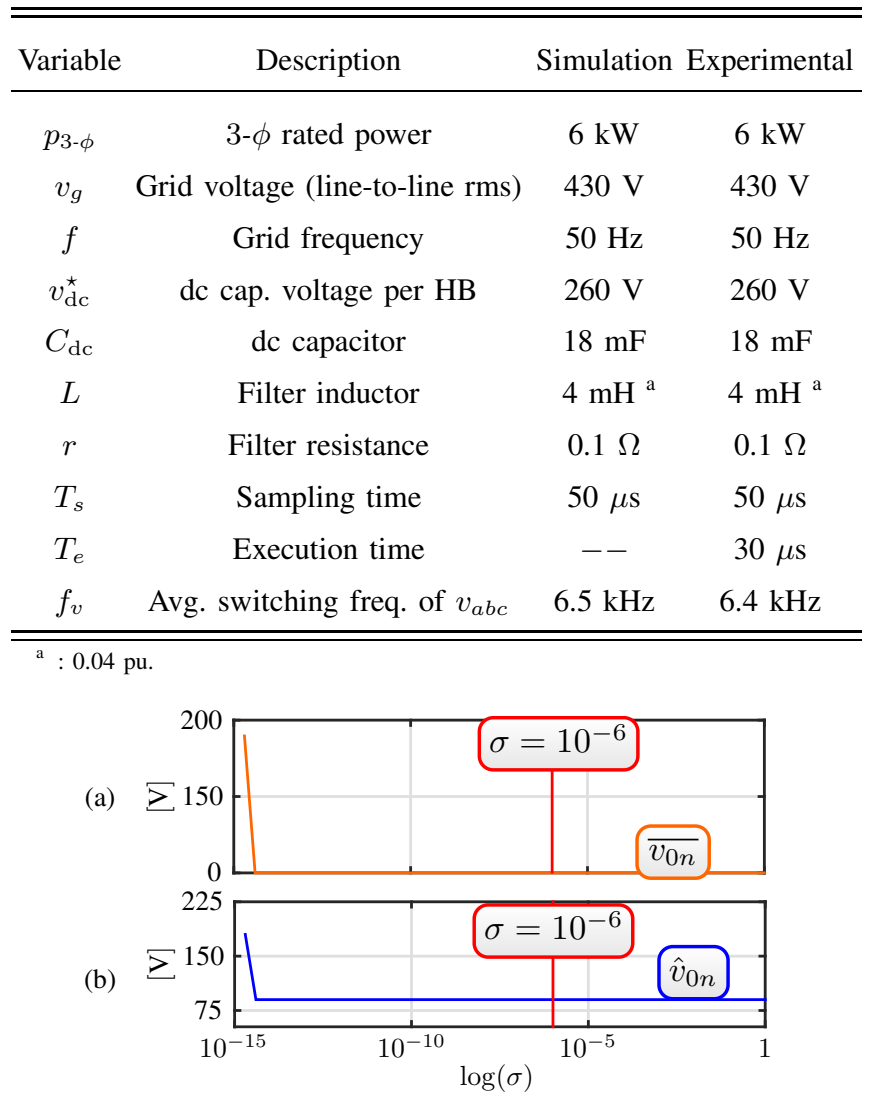

Fig. 3. Simulation-based sensitivity analysis over: (a) average value of $v_{0 n}$ against $\sigma$; (b) common-mode voltage peak value against $\sigma$.

and a minimum peak common-mode voltage, $\hat{v}_{0 n}<90$, is obtained when $\sigma \in\left[10^{-12}, 1\right]$. This gives one a large desing margin without affecting the controller performance in terms of the generated common-mode voltage. However, similarly to a linear quadratic regulator, if $\sigma$ is too large, the controller dynamic performance will be reduced since the second term in the cost function (15) becomes the predominant one. Thus, the converter will tend to apply an input close to its reference, $u(k) \approx u^{\star}(k)$, even during transients. This reduces the closedloop performance of the current tracking error (first term in the cost function (15)). Therefore, the control weighting factor, $\sigma$, is also related to the aggressiveness of the controller (see Section III.C in [24] and Section 3.2.4 in [14]). Consequently, a value of $\sigma=10^{-6}$ was finally chosen.

2) Common-mode voltage minimization: Here, the main results are depicted in Fig. 4. During the first $20 \mathrm{~ms}$, the predictive controller is tuned with $\sigma=0$, which is equivalent to using the standard cost function $J_{\text {std }}(k)$ as per (14). From Fig. 4(c), one can clearly observe that a large average value of the common-mode voltage is obtained. This is also reflected in the CHB phase voltages as shown in Fig. 4(b). To address this issue, at $t=20 \mathrm{~ms}$, the weighting factor is set to $\sigma=10^{-6}$. This immediately reduces the common-mode voltage to a value centered around zero, as shown in Fig. 4(c), yielding a symmetric three-phase CHB voltage, as depicted in Fig. 4(b). It is important to emphasize that in both cases the same line- 
(a)

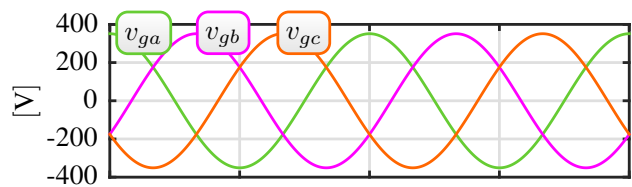

(b)

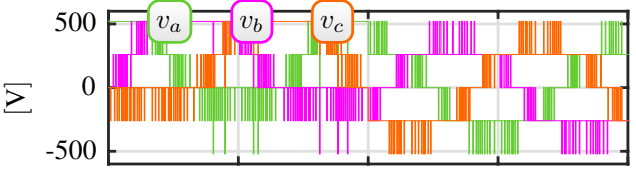

(c)

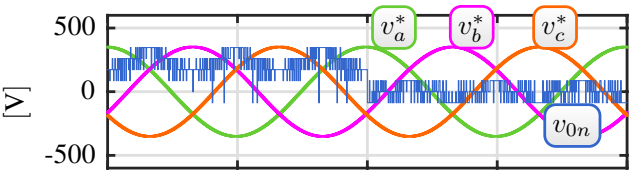

(d)

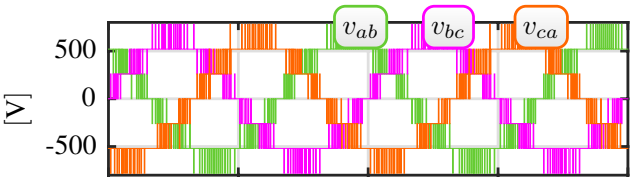

(e)

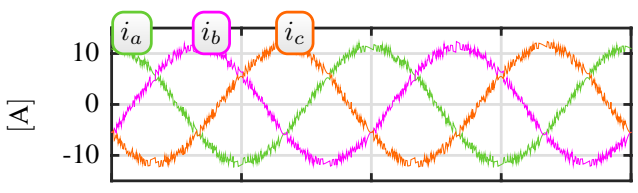

(f)

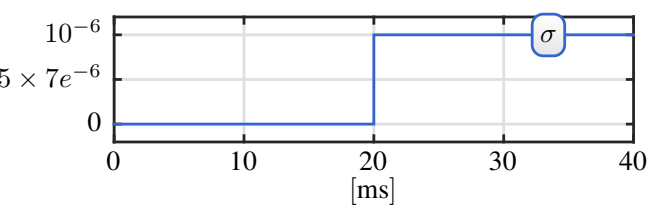

Fig. 4. Simulation results of the proposed FCS-MPC strategy under $v_{0 n}$ minimization. ( $\sigma$ is activated at $t=20 \mathrm{~ms}$ ). (a) grid voltages, (b) converter output voltages, (c) converter output voltage references and common-mode voltage, (d) line-line grid voltage, (e) grid currents and (f) weighting factor.

(a)
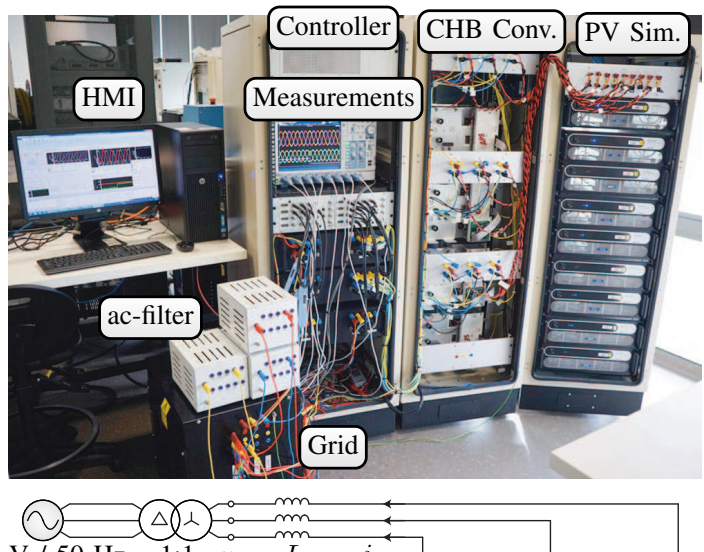

$430 \mathrm{~V} / 50 \mathrm{~Hz} \quad 1: 1 \quad v_{g} \quad L$

(b)

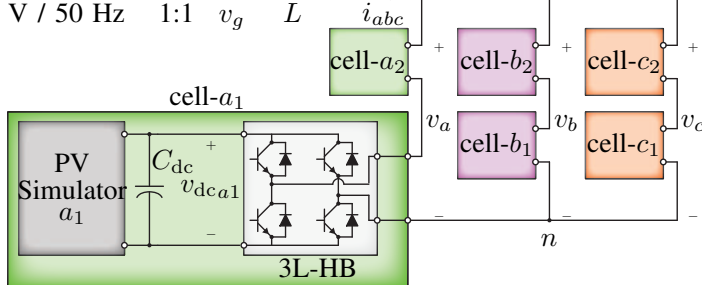

Fig. 5. 6-kVA experimental prototype: (a) hardware and interface (HMI), and (b) schematic diagram.

to-line voltage is obtained (see Fig. 4(d)), which produces the same output currents (see Fig. 4(e)). (a)

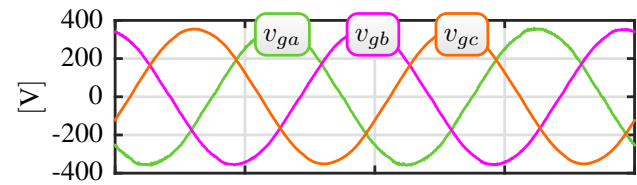

(b)

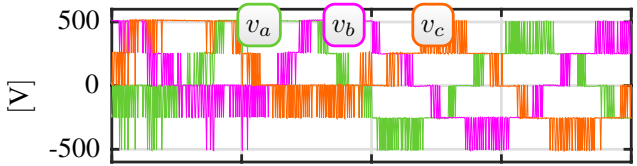

(c)

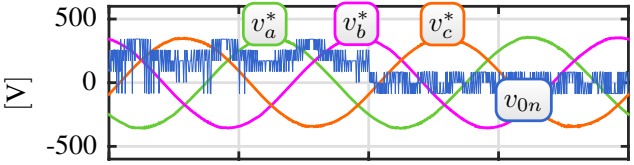

(d)

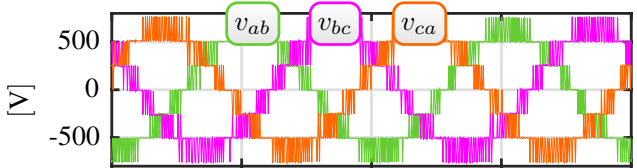

(e)

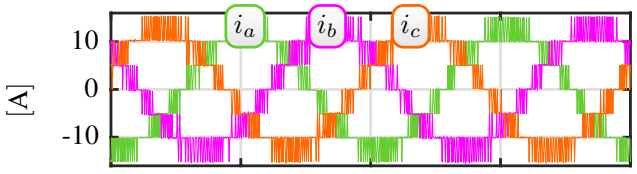

(f)

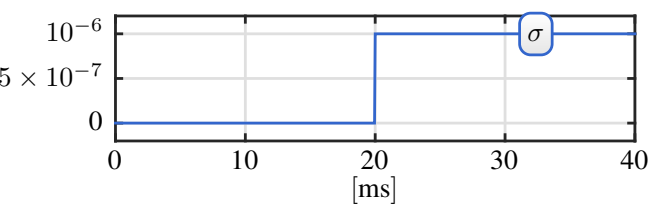

Fig. 6. Experimental results of the proposed FCS-MPC strategy under $v_{0 n}$ minimization. ( $\sigma$ is activated at $t=20 \mathrm{~ms}$ ). (a) grid voltages, (b) converter output voltages, (c) converter output voltage references and common-mode voltage, (d) line-line grid voltage, (e) grid currents and (f) weighting factor.

\section{B. Experimental Results}

The effectiveness of the proposed FCS-MPC strategy under unbalanced power generation among the three phases is tested in a 6kVA three-phase experimental setup (see Fig. 5). Each 3L-HB was fed by an isolated Ametek Elgar TerraSAS PV simulator. The I-V curve of each PV simulator provides a maximum nominal power of $1 \mathrm{~kW}$ at $260 \mathrm{~V}$ for the entire emulated PV array. Since this work is aimed to analyze the proposed controller performance under unbalanced power generation, the dc-dc converters are not included in the labsetup. Therefore, an MPPT stage is no implemented; however, the dc-voltages are still regulated by the power reference generation (see Fig. 2(b)). The overall control strategy was implemented on a dSPACE DS1106 system, where the proposed predictive controller was programmed in C. To account for the linear controllers, a PWM stage to trigger the power switches was implemented in a DS5203 FPGA board. For all cases here analyzed, the common-mode voltage, $v_{0 n}$, was indirectly obtained by adding each measured inverter phase output voltage as per (2). The complete predictive control loop is executed every $50 \mu \mathrm{s}$, while the optimal switching state is computed in $30 \mu \mathrm{s}$. An average switching frequency of $6.4 \mathrm{kHz}$ was obtained for the converter output voltage.

Firstly, the CHB converter is governed by using $\sigma=0$. Figure 6(b) shows that the common-mode voltage presents a 
(a)

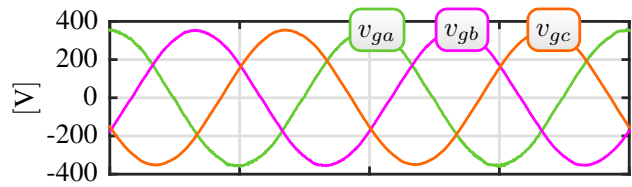

(b)

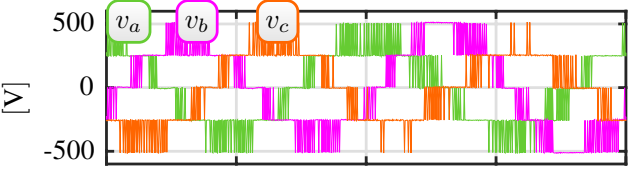

(c)

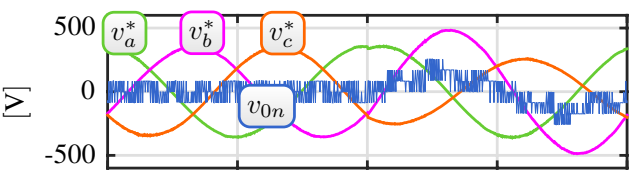

(d)

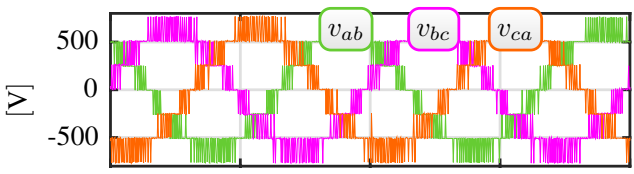

(e)

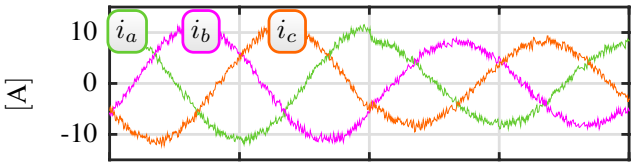

(f)

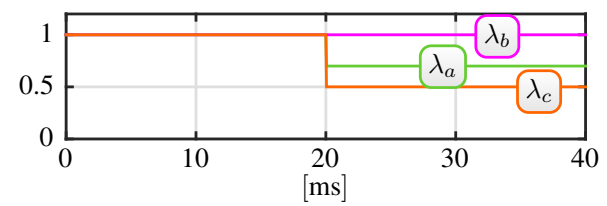

Fig. 7. Experimental performance of the proposed FCS-MPC under unbalanced power generation. (a) grid voltages, (b) converter output voltages, (c) converter output voltage references and common-mode voltage, (d) line-line grid voltage, (e) grid currents and (f) inter-phase power generation ratios.

(a)

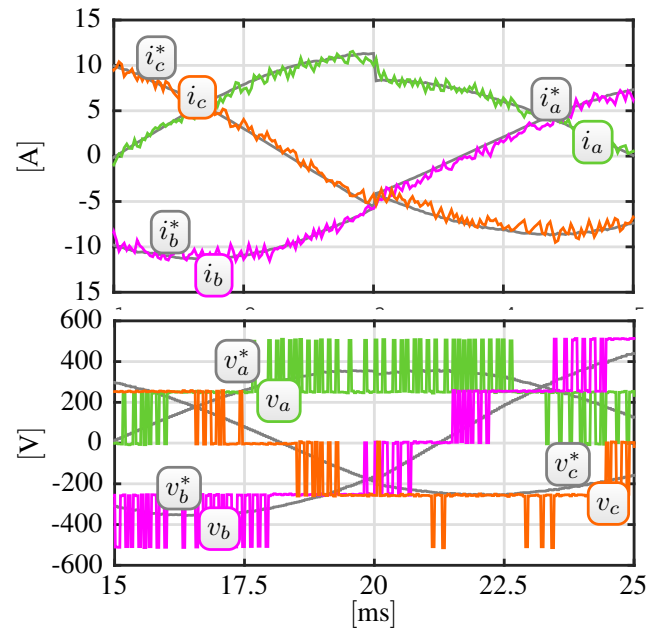

Fig. 8. Zoom of Fig 7 of the proposed FCS-MPC strategy for the unbalanced power generation step change showing (a) $i_{a b c}$; (b) $v_{a b c}$ and their references between 15 and $25 \mathrm{~ms}$.

high value during the first $20 \mathrm{~ms}$. When the weighting factor $\sigma$ is set to $\sigma=10^{-6}$ at $t=20 \mathrm{~ms}, v_{0 n}$ immediately decreases to a value around zero. Thus, the reduction of the commonmode voltage is achieved while maintaining balanced threephase currents as shown in Fig. 6(e).

Figure 7 depicts experimental results obtained under a step change in the dc-source power references from $\lambda_{a}=\lambda_{b}=$ (a)

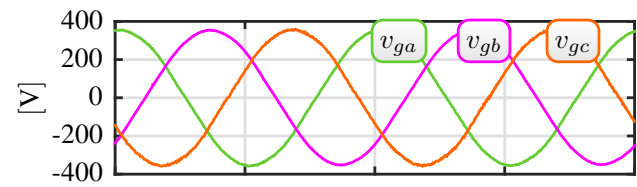

(b)

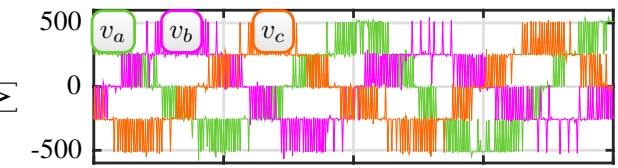

(c)

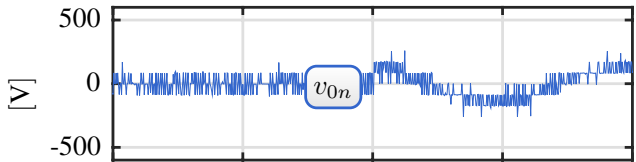

(d)

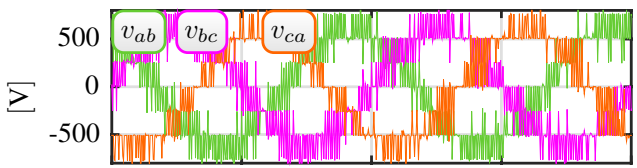

(e)

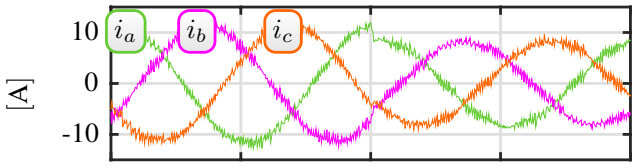

(f)

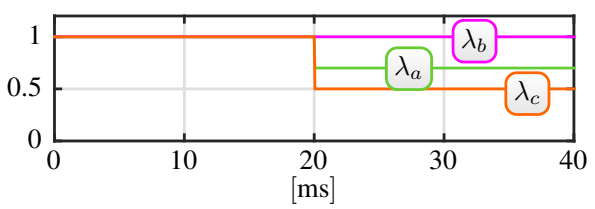

Fig. 9. Experimental performance of the standard VOC strategy under unbalanced power generation. (a) grid voltages, (b) converter output voltages, (c) common-mode voltage, (d) line-line grid voltage, (e) grid currents and (f) inter-phase power generation ratios.

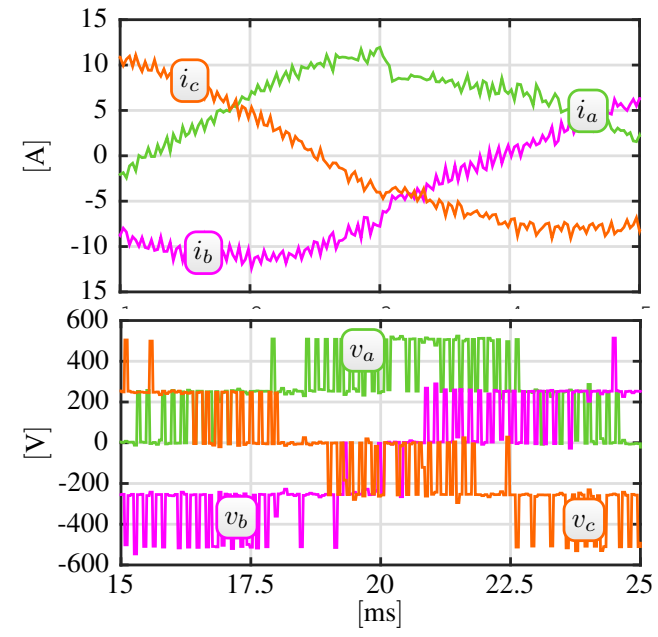

Fig. 10. Zoom of Fig 9 of the standard VOC strategy for the unbalanced power generation step change showing (a) $i_{a b c}$ and (b) $v_{a b c}$ between 15 and $25 \mathrm{~ms}$.

$\lambda_{c}=1$ to $\lambda_{a}=0.7, \lambda_{b}=1$, and $\lambda_{c}=0.5$. Consequently, the grid current changes from maximum power extraction to a peak value proportional to $\frac{\lambda_{a}+\lambda_{b}+\lambda_{c}}{3}$ while maintaining the grid current and the output voltage around their references. In detail, a zoom of the moment when a step change is applied ( $t=20 \mathrm{~ms}$ ) shows that the current injected to the grid quickly achieves its new reference (see Fig. 8(a)). Also, the output 
TABLE ॥

Steady State Performance: Experimental Results

\begin{tabular}{|c|c|c|c|c|c|c|c|c|c|}
\hline \multirow{2}{*}{$\begin{array}{l}\text { Balance case } \\
\text { Weighting factor } \\
\text { Phase }\end{array}$} & \multicolumn{3}{|c|}{$\begin{array}{c}\lambda_{a}=1, \lambda_{b}=1, \lambda_{c}=1 \\
\sigma=0\end{array}$} & \multicolumn{3}{|c|}{$\begin{array}{c}\lambda_{a}=1, \lambda_{b}=1, \lambda_{c}=1 \\
\sigma=1 e^{-6}\end{array}$} & \multicolumn{3}{|c|}{$\begin{array}{c}\lambda_{a}=0.7, \lambda_{b}=1, \lambda_{c}=0.5 \\
\sigma=1 e^{-6}\end{array}$} \\
\hline & $\mathrm{a}$ & $\mathrm{b}$ & $\mathrm{c}$ & $\mathrm{a}$ & $\mathrm{b}$ & $\mathrm{c}$ & $\mathrm{a}$ & $\mathrm{b}$ & $\mathrm{c}$ \\
\hline CHB phase voltage (rms) [V] & 366.0 & 364.2 & 348.9 & 364.5 & 363.2 & 367.2 & 355.6 & 493.2 & 283.5 \\
\hline $\mathrm{THD}_{v}[\%]$ & 21.1 & 21.2 & 22.1 & 13.6 & 13.5 & 13.6 & 14.8 & 10.3 & 17.3 \\
\hline $\mathrm{THD}_{v}(\mathrm{PS}-\mathrm{PWM})[\%]$ & - & - & - & 14.3 & 14.8 & 14.0 & 13.0 & 11.4 & 19.2 \\
\hline Grid current (rms) $[\mathrm{A}]$ & 11.1 & 11.1 & 11.3 & 11.0 & 11.1 & 11.3 & 8.0 & 8.1 & 8.1 \\
\hline $\mathrm{THD}_{i}[\%]$ & 3.1 & 3.3 & 3.3 & 3.0 & 3.1 & 3.5 & 4.6 & 4.6 & 5.0 \\
\hline $\mathrm{THD}_{i}(\mathrm{PS}-\mathrm{PWM})[\%]$ & - & - & - & 2.8 & 2.6 & 3.1 & 4.1 & 3.8 & 4.3 \\
\hline CHB Power [kW] & 2.03 & 2.02 & 1.97 & 2.00 & 2.01 & 2.07 & 1.42 & 1.99 & 1.14 \\
\hline Total Power [kW] & \multicolumn{3}{|c|}{6.02} & \multicolumn{3}{|c|}{6.08} & \multicolumn{3}{|c|}{4.55} \\
\hline
\end{tabular}

voltage responds by tacking its new reference as shown in Fig. 8(b).

The overall performance of the proposed method also relies on the ability to obtain fast dynamic response under transient conditions while maintaining the standard THD values that the aforementioned PWM techniques $(3-5 \%)$ offer in steady state [26]. Thus, the same operating conditions shown in Fig. 7 were replicated using a standard VOC strategy, which is implemented using PI controllers in $d q$-framework combined with PS-PWM. These results are presented in Fig. 9. For further details on the implementation of a standard VOC strategy, the interested reader is refereed to [9] (specifically Fig. 12 in [9]).

As can be clearly appreciated in Fig. 8, the proposed method reaches the desired current reference practically immediately, by applying the optimal output voltage vector that drives the converter currents quickly to their references. In the same way, Fig. 10 shows that the standard VOC strategy is slightly slower when compared to the proposed FCS-MPC strategy. Finally, Table II summarizes the steady-state performance of the proposed controller including $\mathrm{CHB}$ voltages, currents, their respective THDs and power generation per-phase. It is important to emphasize that extracting different amounts of power yields different voltage amplitudes in each phase, which can be higher or lower than its nominal value. Also, in order to tolerate unbalanced cases, normal operation of the converter is designed below the point where THDs are optimal. For those reasons, when a low phase voltage is required, the controller leads the converter to operate in conditions where higher THD values are obtained. The same issues are observed when using linear controllers combined with PS-PWM.

To clearly analyze the closed-loop dynamic response of the proposed predictive controller, a step change in the reactive power (for the balanced case, i.e., $\lambda_{a}=\lambda_{b}=\lambda_{c}=1$ ) is presented in Fig. 11. Here, the reactive power is changed from $-4 \mathrm{kVAR}$ to $4 \mathrm{kVAR}$, while the active power is maintained in $5 \mathrm{~kW}$. One can clearly observe in Fig. 11 that the proposed FCS-MPC provides a faster closed-loop dynamic when compared to the standard VOC strategy. Moreover, the predictive controller presents a flat response while the PI (a)

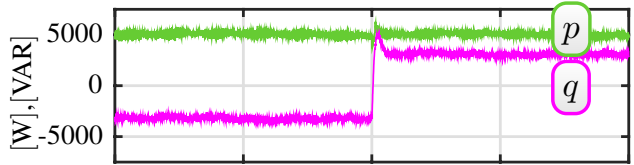

(b)

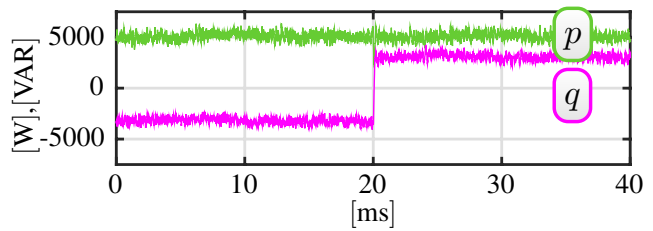

Fig. 11. Experimental performance of (a) standard VOC strategy and (b) proposed FCS-MPC strategy under reactive power flow direction step change.

controller exhibits an overshoot. To reduce this overshoot, one can decrease the controller bandwidth; however, this will lead to a slower closed-loop dynamic.

Therefore, as evidenced by the experimental results, both the proposed predictive control strategy and the standard VOC strategy offer similar steady-state performance in terms THD. However, the proposed FCS-MPC outperforms PWM-based PI controllers when larger active and/or reactive power transients are required; see also [21].

\section{CONClusion}

In this work, a predictive control strategy for $\mathrm{CHB}$ converters has been proposed. This approach is able to extract unbalanced power from each $\mathrm{CHB}$ converter phase while injecting balanced power to the grid. The key novelty of this proposal is that the unbalanced power generation issue is explicitly considered into the optimal control problem by including an input tracking error in the proposed cost function. The resulting control scheme presents a simple structure with a lower number of cascaded control loops are required. Moreover, the proper selection of the weighting factor allows one to preserve the well-known fast dynamic response of the predictive controller during transient conditions and achieve the power balance in steady state, as verified by the simulation and experimental results. 
The proposed solution (input tracking reference) can be easily added to existing FCS-MPC embodiments which opens the door to include interesting targets for grid-connected converters for PV applications such as inter-cell power balance, reactive power compensation, low voltage ride-through capability, etc.

\section{REFERENCES}

[1] S. Kouro, M. Malinowski, K. Gopakumar, J. Pou, L. Franquelo, B. Wu, J. Rodríguez, M. A. Pérez, and J. Leon, "Recent Advances and Industrial Applications of Multilevel Converters," IEEE Trans. Ind. Electron., vol. 57, DOI 10.1109/TIE.2010.2049719, no. 8, pp. 2553-2580, Aug. 2010.

[2] E. Al-nabi, B. Wu, N. Zargari, and V. Sood, "Input Power Factor Compensation for High-Power CSC Fed PMSM Drive Using d Axis Stator Current Control," IEEE Trans. Ind. Electron., vol. 59, DOI 10.1109/TIE.2011.2158772, no. 2, pp. 752-761, Feb. 2012.

[3] P. Acuna, L. Moran, M. Rivera, R. P. Aguilera, R. Burgos, and V. G. Agelidis, "A Single-Objective Predictive Control Method for a Multivariable Single-Phase Three-Level NPC ConverterBased Active Power Filter," IEEE Trans. Ind. Electron., vol. 62, DOI 10.1109/TIE.2015.2393556, no. 7, pp. 4598-4607, Jul. 2015.

[4] J. Carrasco, L. Franquelo, J. Bialasiewicz, E. Galvan, R. Guisado, M. Prats, J. Leon, and N. Moreno-Alfonso, "Power-Electronic Systems for the Grid Integration of Renewable Energy Sources: A Survey," IEEE Trans. Ind. Electron., vol. 53, DOI 10.1109/TIE.2006.878356, no. 4, pp. 1002-1016, Jun. 2006.

[5] R. P. Aguilera, P. Lezana, and D. E. Quevedo, "Switched Model Predictive Control for Improved Transient and Steady-State Performan ce," IEEE Trans. Ind. Informat., vol. 11, DOI 10.1109/TII.2015.2449992, no. 4, pp. 968-977, Aug. 2015.

[6] Y. Yu, G. Konstantinou, B. Hredzak, and V. G. Agelidis, "Power Balance of Cascaded H-Bridge Multilevel Converters for LargeScale Photovoltaic Integration," IEEE Trans. Power Electron., vol. 31, DOI 10.1109/TPEL.2015.2406315, no. 1, pp. 292-303, Jan. 2016.

[7] M. A. Pérez, S. Bernet, J. Rodríguez, S. Kouro, and R. Lizana, "Circuit Topologies, Modeling, Control Schemes, and Applications of Modular Multilevel Converters," IEEE Trans. Power Electron., vol. 30, DOI 10.1109/TPEL.2014.2310127, no. 1, pp. 4-17, Jan. 2015.

[8] S. Rivera, S. Kouro, B. Wu, J. Leon, J. Rodríguez, and L. Franquelo, "Cascaded H-bridge multilevel converter multistring topology for large scale photovoltaic systems," in IEEE 20th International Symposium on Industrial Electronics (ISIE), DOI 10.1109/ISIE.2011.5984437, Jun. 2011, pp. 1837-1844.

[9] Y. Yu, G. Konstantinou, B. Hredzak, and V. G. Agelidis, "Power Balance Optimization of Cascaded H-Bridge Multilevel Converters for LargeScale Photovoltaic Integration," IEEE Trans. Power Electron., vol. 31, DOI 10.1109/TPEL.2015.2407884, no. 2, pp. 1108-1120, Feb. 2016.

[10] P. Sochor and H. Akagi, "Theoretical comparison in energy-balancing capability between star- and delta-configured modular multilevel cascade inverters for utility-scale photovoltaic systems," IEEE Trans. Power Electron., vol. 31, DOI 10.1109/TPEL.2015.2442261, no. 3, pp. 19801992, Mar. 2016.

[11] J. Rodríguez, M. P. Kazmierkowski, J. Espinoza, P. Zanchetta, H. AbuRub, H. A. Young, and C. A. Rojas, "State of the Art of Finite Control Set Model Predictive Control in Power Electronics," IEEE Trans. Ind. Informat., vol. 9, DOI 10.1109/TII.2012.2221469, no. 2, pp. 1003-1016, May. 2013.

[12] S. Vazquez, J. Leon, L. Franquelo, J. Rodríguez, H. A. Young, A. Marquez, and P. Zanchetta, "Model Predictive Control: A Review of Its Applications in Power Electronics," IEEE Ind. Electron. Mag., vol. 8, DOI 10.1109/MIE.2013.2290138, no. 1, pp. 16-31, Mar. 2014.

[13] S. Vazquez, A. Marquez, R. Aguilera, D. Quevedo, J. Leon, and L. Franquelo, "Predictive Optimal Switching Sequence Direct Power Control for Grid-Connected Power Converters," IEEE Trans. Ind. Electron., vol. 62, DOI 10.1109/TIE.2014.2351378, no. 4, pp. 2010-2020, Apr. 2015.

[14] D. E. Quevedo, R. P. Aguilera, and T. Geyer, "Predictive Control in Power Electronics and Drives: Basic Concepts, Theory, and Methods," in Advanced and Intelligent Control in Power Electronics and Drives. New York, NY, USA: Springer, 2014, vol. 531, pp. 181-226.

[15] P. Lezana, R. P. Aguilera, and D. E. Quevedo, "Model Predictive Control of an Asymmetric Flying Capacitor Converter," IEEE Trans. Ind. Electron., vol. 56, DOI 10.1109/TIE.2008.2007545, no. 6, pp. 18391846, Jun. 2009.
[16] R. P. Aguilera, P. Lezana, and D. E. Quevedo, "Finite-Control-Set Model Predictive Control With Improved Steady-State Performance," IEEE Trans. Ind. Informat., vol. 9, DOI 10.1109/TII.2012.2211027, no. 2, pp. 658-667, May. 2013.

[17] J. D. Barros, J. F. A. Silva, and E. G. A. Jesus, "Fast-Predictive Optimal Control of NPC Multilevel Converters," IEEE Trans. Ind. Electron., vol. 60, DOI 10.1109/TIE.2012.2206352, no. 2, pp. 619-627, Feb. 2013.

[18] V. Yaramasu, M. Rivera, M. Narimani, B. Wu, and J. Rodríguez, "Model Predictive Approach for a Simple and Effective Load Voltage Control of Four-Leg Inverter With an Output LC Filter," IEEE Trans. Ind. Electron., vol. 61, DOI 10.1109/TIE.2013.2297291, no. 10, pp. 52595270, Oct. 2014.

[19] P. Cortés, J. Rodríguez, P. Antoniewicz, and M. Kazmierkowski, "Direct Power Control of an AFE Using Predictive Control," IEEE Trans. Power Electron., vol. 23, DOI 10.1109/TPEL.2008.2002065, no. 5, pp. 25162523, Sep. 2008.

[20] D. E. Quevedo, R. P. Aguilera, M. A. Pérez, P. Cortés, and R. Lizana, "Model Predictive Control of an AFE Rectifier With Dynamic References," IEEE Trans. Power Electron., vol. 27, DOI 10.1109/TPEL.2011.2179672, no. 7, pp. 3128-3136, Jul. 2012.

[21] A. Calle-Prado, S. Alepuz, J. Bordonau, J. Nicolas-Apruzzese, P. Cortés, and J. Rodríguez, "Model Predictive Current Control of GridConnected Neutral-Point-Clamped Converters to Meet Low-Voltage Ride-Through Requirements," IEEE Trans. Ind. Electron., vol. 62, DOI 10.1109/TIE.2014.2364459, no. 3, pp. 1503-1514, Mar. 2015.

[22] C. Buccella, C. Cecati, and H. Latafat, "Digital Control of Power Converters - A Survey," IEEE Trans. Ind. Informat., vol. 8, DOI 10.1109/TII.2012.2192280, no. 3, pp. 437-447, Aug. 2012.

[23] T. J. Vyncke, S. Thielemans, and J. A. Melkebeek, "Finite-Set ModelBased Predictive Control for Flying-Capacitor Converters: Cost Function Design and Efficient FPGA Implementation," IEEE Trans. Ind. Informat., vol. 9, DOI 10.1109/TII.2012.2223707, no. 2, pp. 1113-1121, May. 2013.

[24] R. P. Aguilera and D. E. Quevedo, "Predictive Control of Power Converters: Designs With Guaranteed Performance," IEEE Trans. Ind. Informat., vol. 11, DOI 10.1109/TII.2014.2363933, no. 1, pp. 53-63, Feb. 2015.

[25] P. Cortés, A. Wilson, S. Kouro, J. Rodríguez, and Abu-Rub, "Model Predictive Control of Multilevel Cascaded H-Bridge Inverters," IEEE Trans. Ind. Electron., vol. 57, DOI 10.1109/TIE.2010.2041733, no. 8, pp. 2691-2699, Aug. 2010.

[26] C. D. Townsend, T. Summers, and R. E. Betz, "Phase-Shifted Carrier Modulation Techniques for Cascaded H-Bridge Multilevel Converters," IEEE Trans. Ind. Electron., vol. 62, DOI 10.1109/TIE.2015.2442516, no. 11, pp. 6684-6696, Nov. 2015.

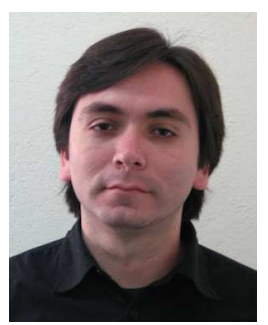

Ricardo P. Aguilera (S'01-M12) received his M.Sc. degree in Electronics Engineering from the Universidad Tecnica Federico Santa Maria (UTFSM), Chile, 2007, and Ph.D. degree in Electrical Engineering from The University of Newcastle (UN), Australia, 2012. From 2012 to 2013, he was a Research Academic at UN where he was part of the Centre for Complex Dynamic Systems and Control. From 2014 to 2016, he was a Senior Research Associate at The University of New South Wales (UNSW) Australia, where he was part of the Australian Energy Research Institute (AERI). Since 2016, he joined The School of Electrical, Mechanical and Mechatronic Systems, at The University of Technology Sydney (UTS), Australia, where he currently holds a Lecturer position. His main research interests include power electronics, and theoretical and practical aspects on model predictive control.

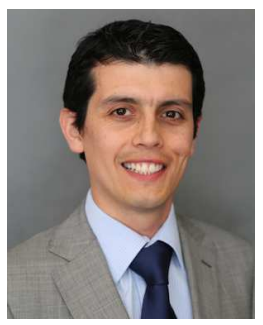

Pablo Acuna (M'12) received the B.Sc. in Electronics Engineering, the Electronics Engineering Professional, and the Ph.D. degrees in Electrical Engineering from the University of Concepcion, Chile, in 2004, 2007, and 2013 respectively. He is currently Research Associate at University of New South Wales, Australia. His research interests include electrical power conversion systems and its applications to industry, transportation and utility. 


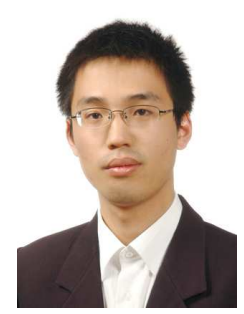

Yifan Yu (S13) received the B.Eng. degree in electrical engineering from the Harbin Institute of Technology, Harbin, China, in 2010. He is currently working toward the Ph.D. degree at the University of New South Wales, Sydney, N.S.W., Australia. His research interests include multilevel converters, Z-Source inverters, photovoltaic grid connection, and other aspects of power electronic technology for renewable energy sources.

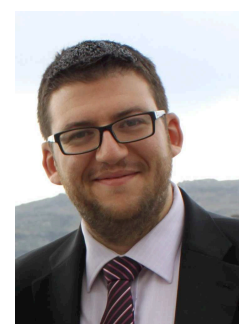

Georgios Konstantinou (S'08-M'11) received the B.Eng. degree in electrical and computer engineering from the Aristotle University of Thessaloniki, Thessaloniki, Greece, in 2007 and the Ph.D. degree in electrical engineering from UNSW Australia, Sydney, in 2012. He is currently a Lecturer with the School of Electrical Engineering and Telecommunications at UNSW Australia. His research interests include multilevel power electronics converters and their applications, pulse width modulation and selective harmonic elimination techniques.

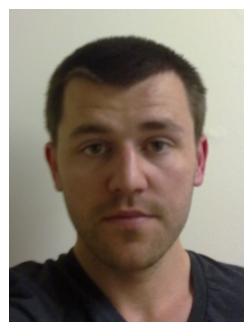

Christopher D. Townsend (S'09-M'13) received the B.E. (2009) and Ph.D. (2013) degrees in electrical engineering from the University of Newcastle, Australia. He has spent the last four years working at ABB Corporate Research, Sweden and then as a senior research associate at the Australian Energy Research Institute, University of New South Wales. He is currently working as a post-doctoral researcher at the University of Newcastle, Australia. His current research interests include topologies and modulation strategies for multi-level converters. He is a member of the Power Electronics and Industrial Electronics Societies of the IEEE.

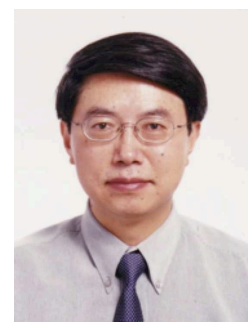

Bin Wu (S'89-M'92-SM'99-F'08) received his M.A.Sc. and Ph.D. degrees in electrical and computer engineering from the University of Toronto, Canada in 1989 and 1993, respectively. He joined Ryerson University in 1993 where he is currently a Professor and $\mathrm{Se}$ nior NSERC/Rockwell Automation Industrial Research Chair in Power Electronics and Electric Drives. Dr. Wu has published more than 350 technical papers, authored/coauthored two Wiley-IEEE Press books, and holds more than $30 \mathrm{granted} /$ pending US/European patents in the area of power conversion, medium voltage drives, and renewable energy systems.

Dr. Wu received the Gold Medal of the Governor General of Canada in 1993, Premiers Research Excellence Award in 2001, NSERC Synergy Award for Innovation in 2002, Ryerson Distinguished Scholar Award in 2003, Ryerson YSGS Outstanding Contribution to Graduate Education Award and Professional Engineers Ontario (PEO) Engineering Excellence Medal in 2014. He is a fellow of Engineering Institute of Canada (EIC) and Canadian Academy of Engineering (CAE).

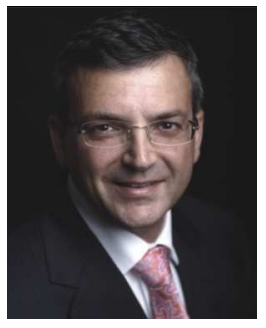

Vasssilios G. Agelidis (SM'00-F'16) received the B.Eng. degree in Electrical Engineering from the Democritus University of Thrace, Greece, in 1988, the M.S. degree in Applied Science from Concordia University, Montreal, QC, Canada, in 1992, and the Ph.D. degree in Electrical Engineering from Curtin University, Perth, Australia, in 1997. He has done extensive research in the field of smarter grid infrastructure and sustainable energy systems incorporating solar and wind energy sources. He is currently Professor of Power Engineering at the School of Electrical Engineering and Telecomunications at the University of New South Wales (UNSW). 